\title{
ARQUITECTURA DE CONTROL HÍBRIDA PARA LA NAVEGACIÓN DE VEHÍCULOS SUBMARINOS NO TRIPULADOS
}

\author{
Francisco J. Lastra $^{(a)}$, Jesús A. Trujillo ${ }^{(b)}$, Francisco J. Velasco ${ }^{(a)}$, Elías Revestido ${ }^{(a)}$. \\ (a) C/ Gamazo 1, E. T. S. de Náutica, 39004, Santander, Universidad de Cantabria, \\ e-mail: fjlastra@teisa.unican.es, velascof@unican.es, revestidoe@unican.es. \\ (b) C/Avda. de Fuencarral, 44, Madrid, Iberalter, e-mail:info@iberalter.es.
}

\section{Resumen}

En el presente trabajo, se propone una arquitectura hibrida basada en sistemas multi-agentes en capas. Esta arquitectura presenta cualidades de modularidad y escalabilidad. También se desarrolla una metodología para el seguimiento de trayectorias basado en la generación de un espacio virtual fiable, en el que la navegación se lleva a cabo en condiciones operativas de seguridad y eficacia. Se espera obtener una reducción en el tiempo de procesamiento de operaciones durante la inspección.

Palabras clave: Arquitectura de control híbrida, modularidad, escalabilidad, sistemas supervisores.

\section{Introducción}

Los sistemas subacuáticos autónomos no tripulados han atesorado un aumento en su autonomía durante la última década [6], tanto desde el punto de vista del suministro de energía como del punto de vista de la capacidad de decisión. Esta autonomía permite la posibilidad de realizar aplicaciones fiables y seguras con este tipo de sistemas.

La inclusión de sistemas robotizados en el entorno subacuático es muy compleja, principalmente debido a un comportamiento dinámico variable e impredecible del entorno que tiene un impacto significativo en las diferentes actividades que pueden llevarse a cabo. Una de estas actividades complejas, es la inspección de cables submarinos [7, 2], debido a la dificultad de operar en un ambiente hóstil. Esto constituye una línea de investigación de interés creciente.

Los sistemas de inspección para cables submarinos han evolucionado con el desarrollo de sistemas robóticos bajo el agua. La tendencia operativa submarina convencional se puede dividir en dos grupos: uno es la inspección submarina del cable por buceadores y otro es la inspección usando vehículos submarinos autónomos. El primero es el más arriesgado, ya que los buzos están expuestos a los peligros inherentes a las altas profundidades. Este último se realiza mediante el uso de vehículos submarinos que ayudan a un operador humano en una operación peligrosa. Básicamente, los vehículos submarinos [13] desarrollan tareas de localización y posicionamiento, con el fin de mejorar la inspección de cables submarinos a través de sistemas de visión bajo el agua (visión estéreo).

Debido a la alta complejidad de estas aplicaciones es esencial, su desarrollo bajo el amparo una arquitectura de control que contiene implícitamente características de modularidad y escalabilidad, que permiten transacciones entre niveles de arquitectura. Hoy en día los sistemas autónomos no tripulados tienen altas restricciones para realizar estas acciones de inspección. Estas restricciones se refieren al tiempo de ejecución de las rutinas de procesamiento de imágenes, bloques de exploración, etc.

En este trabajo se propone una arquitectura cooperativa multi-agente $[8,12,11]$ que deriva del concepto convencional de arquitectura híbrida basado en un sistema estratificado dividido en grupos comportamentales: un comportamiento reactivo, en el que el sistema debe reaccionar de manera automática a un evento particular, es decir, si se detecta un objeto, el sistema de evitación de obstáculos modifica la trayectoria del vehículo para evitar un impacto y un comportamiento deliberativo, en el que se evalúan diferentes posibilidades y se seleccionan las más adecuadas en cada situación, por ejemplo, la generación de un camino entre dos puntos dependiendo del consumo, etc.

\section{El Problema}

Como se ha mencionado anteriormente, el objetivo de este trabajo es la inspección de cables submarinos mediante sistemas autónomos no tripulados. Para ello, el vehículo debe rastrear el cable y evaluar los datos capturados por la instrumentación para determinar los defectos del cable.

La metodología convencional para la inspección de cables submarinos consiste en un seguidor de cables, en el fondo del mar, basado en datos adquiridos de un sonar frontal. Para ello, las empresas utilizan vehículos submarinos no tripulados que detectan el cable con antelación y gen- 
eran la trayectoria de referencia del vehículo. Sin embargo, en nuestra propuesta, generamos una trayectoria virtual basada en datos de sonar de barrido lateral que predice la trayectoria virtual por medio de datos previos. De esta manera, proponemos una arquitectura que se ocupa del seguimiento de cables submarinos utilizando datos de sonar de barrido lateral.

La estructura interna de los cables submarinos suele incorporar diferentes tipos de cables de acero que evitan cambios abruptos en la dirección del cable que descansa sobre el lecho marino. Es por eso que se espera que las trayectorias de estos cables sean suaves y que este aspecto se tenga en cuenta en el diseño del seguidor en las secciones siguientes.

\subsection{El Vehículos Subacuático}

En el presente trabajo, para resolver el problema indicado, se utiliza un vehículo con forma de torpedo, en el que se montan dos tipos diferentes de sonar: un sonar de barrido lateral (SBL) [3] y un sonar de navegación (SN). De esta manera, el vehículo puede adquirir datos relacionados con el lecho marino, en ambos lados del vehículo. La cobertura SBL nos permite detectar aproximadamente 1 metro del ancho del lecho marino. El SN está situado en la proa del vehículo y el SBL en la parte central del vehículo.

El estudio de la imagen se realiza mediante técnicas de visión por ordenador. Gracias a la imagen SN del entorno, es posible detectar objetos con anticipación, tanto dinámicos como estáticos, proporcionando un camino fiable y seguro. Es probable que en la imagen del SBL aparezcan diferentes objetos sobre el lecho marino, en ese estudio se debe tener en cuenta el estudio de las sombras acústicas, ya que proporciona características de los objetos.

Como se ha mencionado anteriormente, el SBL esta situado en la parte central del vehículo proporcionando información de objetos dentro de la zona determinada por el ángulo sonar de detección. Esta zona de detección depende de la profundidad, por lo tanto hay límites más altos y más bajos para la detección fiable de cables submarinos.

Después de analizar y detectar el cable utilizando el SBL, los nuevos puntos de posición que corroboran la trayectoria correcta se determinan mediante un bloque de navegación que se describirá en la sección 3. Por lo tanto, el vehículo navega a partir del desarrollo teórico de una trayectoria obtenida ajustando, los puntos SBL adquiridos, por una función. Es importante destacar la ex-

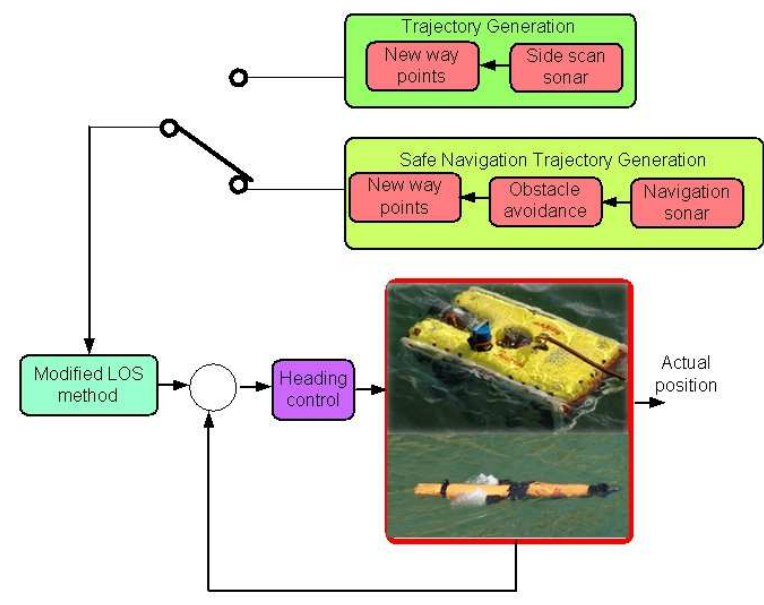

Figura 1: Arquitectura básica para el control de vehículos subacuáticos.

istencia de una relación entre la generación de la trayectoria y los cambios de velocidad repentinos en el equipo, ya que estos nuevos puntos de generación dependen de la velocidad del vehículo y por lo tanto de la posición obtenida en cada iteración.

\subsection{Arquitectura Básica}

La figura 1 muestra la arquitectura básica para el seguimiento de cables submarinos utilizando un vehículo con forma de torpedo con las características indicadas anteriormente. Esta arquitectura se basa en 4 módulos. Un módulo de generación de trayectorias para el seguimiento del cable submarino, que es capaz de generar una trayectoria teórica desde una función que combina todos los puntos previamente adquiridos para el siguiente conjunto de puntos de referencia, un sistema de navegación seguro, capaz de analizar y evitar cualquier objeto que pueda interceptar la trayectoria establecida por el módulo anterior, un sistema de decisión, basado en las reglas ECA (eventocondición-acción) [1], capaz de modificar el comportamiento del sistema cambiando el modo operativo de navegación a navegación segura en el instante que se detecta un peligro potencial y, finalmente, un sistema de seguimiento trayectoria, para seguir fielmente cada uno de los puntos de ruta proporcionados por el módulo de navegación encargado de reducir el error transversal.

\section{Arquitectura de Control Propuesta}

A partir de la arquitectura básica mencionada, se ha desarrollado una arquitectura compleja basada en un marco de sistemas multi-agente. La figura 2 muestra los diferentes módulos enmarcados den- 


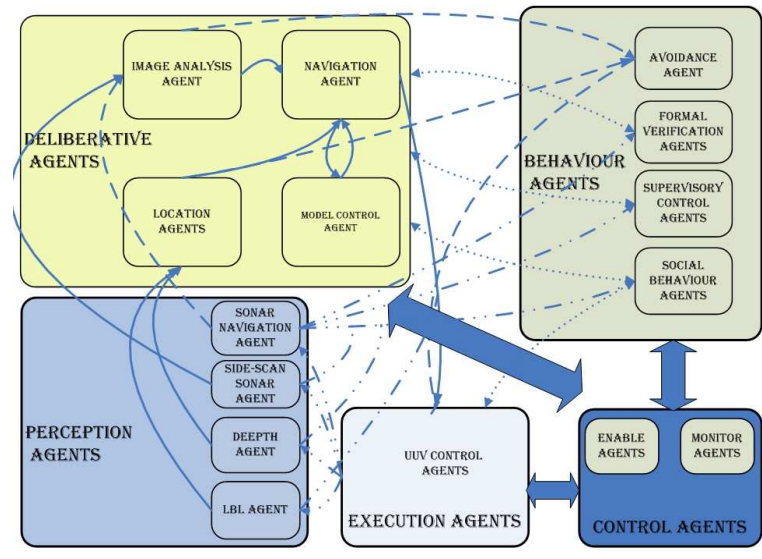

Figura 2: Arquitectura multi-agentes.

tro de cada capa y sus agentes correspondientes. Hay diferentes clases de agentes responsables de diferentes tareas, un grupo de ellos son responsables de la adquisición de datos ambientales y de posición del vehículo. Otros están a cargo de controlar el comportamiento dinámico del vehículo en diferentes situaciones. Por último, hay un grupo de ellos que proporciona cohesión a los diferentes módulos, los agentes de supervisión, cuya funcionalidad es unir a todos los grupos, mediante la planificación y control de los diferentes bloques.

Se ha incluido un bloque de control de agentes para el correcto funcionamiento del sistema, que habilita/deshabilita los módulos en función del rendimiento general del sistema. Es decir, el bloque de control del supervisor [10] modifica el rendimiento general con el fin de evitar comportamientos no deseados. Un claro ejemplo es la desactivación del bloque de navegación cuando el vehículo está dentro del bloque de evitación de obstáculos.

La figura 3 muestra los módulos y submódulos que componen el sistema, junto con los datos que se transmiten entre ellos. Como se ha indicado anteriormente, la arquitectura propuesta está dispuesta en capas, por lo tanto, estos módulos deben estar situados en su capa correcta.

La capa deliberativa tiene los módulos de navegación, los módulos de pérdida de cable. Por otra parte en la capa reactiva, podemos encontrar los módulos de evitación de obstáculos y los módulos de seguimiento de trayectoria.

El sonar del módulo de adquisición y procesamiento de imágenes es común a ambas capas, ya que los datos obtenidos por el SBL se usan en la capa deliberativa mientras que el SN es alimentado por sus propios datos.

\subsection{Módulos}

La arquitectura propuesta consta de cinco módulos principales. Las restricciones de tiempo de los módulos son diferentes, mientras que algunas de ellas funcionan en tiempo real como lo hace el módulo de ejecución, otros sistemas están latentes esperando su habilitación de un evento, el evento de este último tipo son los módulos de Evitación de Obstáculos y los Módulos de Pérdida de Cable. Esta sección resume cada uno de los módulos:

El módulo de navegación es responsable de la generación de una ruta fiable y segura para el vehículo. En el módulo de adquisición y procesamiento de imágenes se obtiene la posición del cable y se transfiere al sistema de coordenadas fijado a tierra. El sistema genera una trayectoria a partir de los puntos adquiridos en las etapas anteriores, para ello, los datos obtenidos se utilizan de tal manera que el sistema de decisión no sigue el camino definido cuando hay una gran variación en la velocidad. Cuando se ha definido la trayectoria del cable, que es una función de velocidad, se genera una trayectoria factible para el vehículo al alisar la trayectoria mediante b-splines. La zona de detección de objetos se calcula a partir de la definición de B-spline [9]. Esto se puede hacer resolviendo la siguiente ecuación:

$$
P(t)=\sum_{i=1}^{n} P_{i} N_{i, j}(t)
$$

siendo $P(t)$ la posición del vector a lo largo de una curva de dimensión $m$ como una función de $t \epsilon \Re$, una curva de tipo spline de orden $j, P_{i} \epsilon \Re^{m}(i=$ $0, \ldots, n)$ puntos de control y $N_{i, j}(t)$ son las funciones normalizadas B-spline de orden $j$ y el vector $\Xi=\left\{x_{0}, \ldots x_{n+k}\right\}$. Estas funciones B-spline están definidas por las fórmulas de recursión de Cox-de Boor,

$$
N_{i, j} \equiv\left\{\begin{array}{l}
1 \text { if } x_{i} \leq t \leq x_{i+1} \\
0 \text { for the rest of the cases }
\end{array}\right.
$$

y para todo $j>1$

$N_{i, j}(t)=\frac{\left(t-x_{i}\right) N_{i, j-1}(t)}{x_{i+j-1}-x_{i}}+\frac{\left(-t+x_{i+j}\right) N_{i+1, j-1}(t)}{x_{i+j}-x_{j+1}}$

donde $t$ es el tiempo, $x$ es la posición del vehículo en el eje horizontal.

Aplicando las expresiones (1), (2) y (3), obtenemos una zona de operación segura como se puede ver en la figura 4.

De esta manera, a partir de la zona citada podemos establecer un volumen seguro para la operación del sistema (Figura 5). Sobreponiendo las 


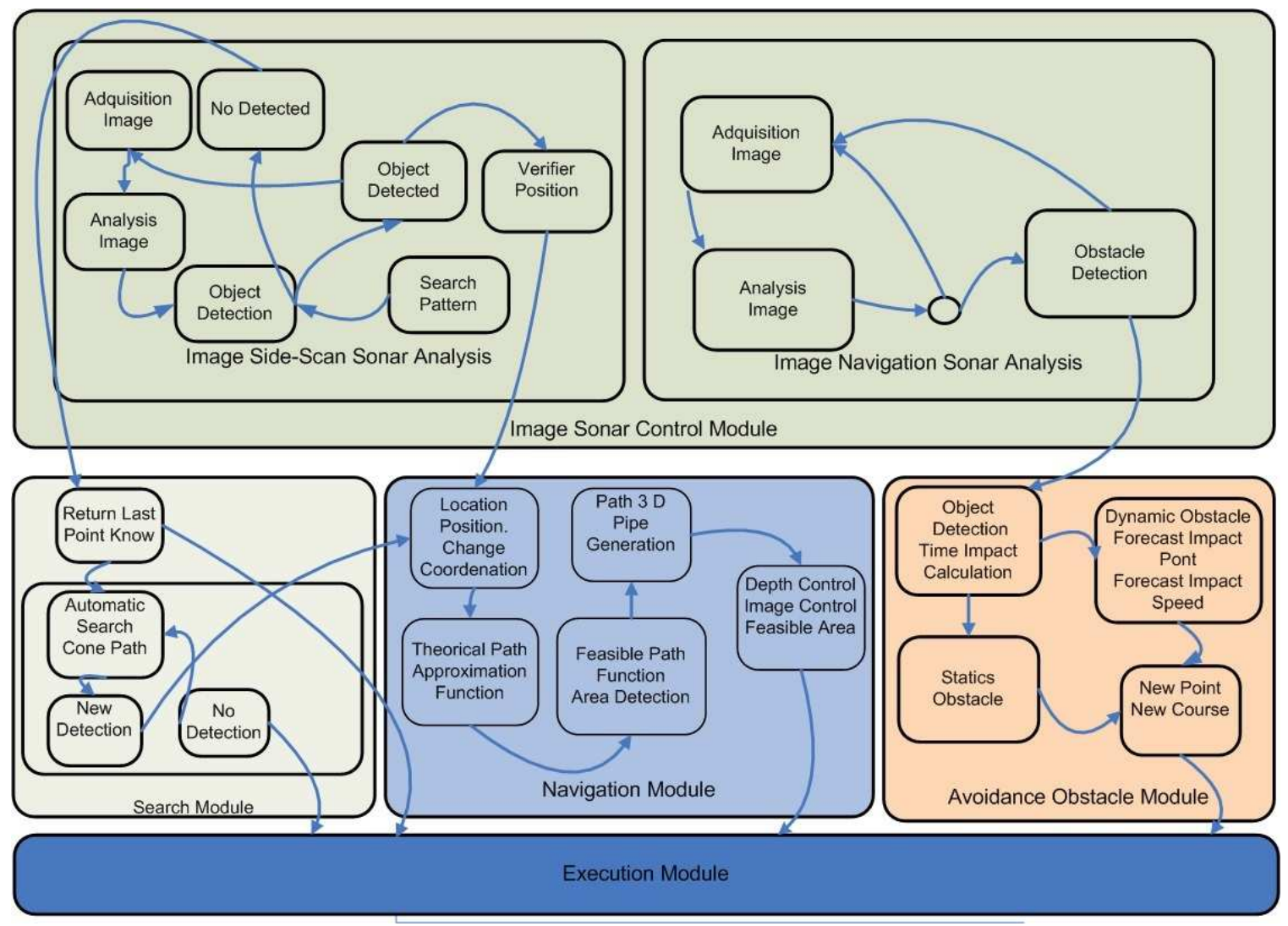

Figura 3: Arquitectura de control propuesta.

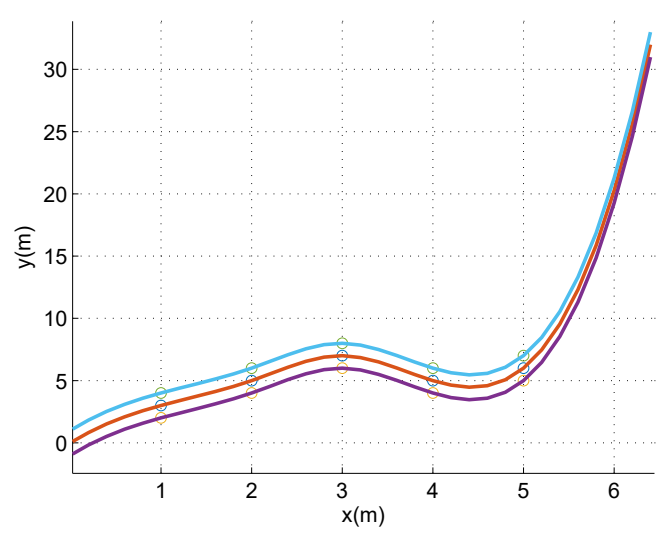

Figura 4: Área de detección (área entre las líneas azul y morada) y ruta teórica (línea roja)

diferentes capas, limitadas sólo por la profundidad y la caracterización precisa de los objetos del fondo marino.

El módulo de control de imagen de sonar, alimenta los módulos de navegación y evitación de obstáculos. Al estudiar las imágenes SBL, se determina la posición del cable en el lecho marino. Además, al estudiar las imágenes del SN se determina la existencia de objetos en la trayectoria del sistema robótico.

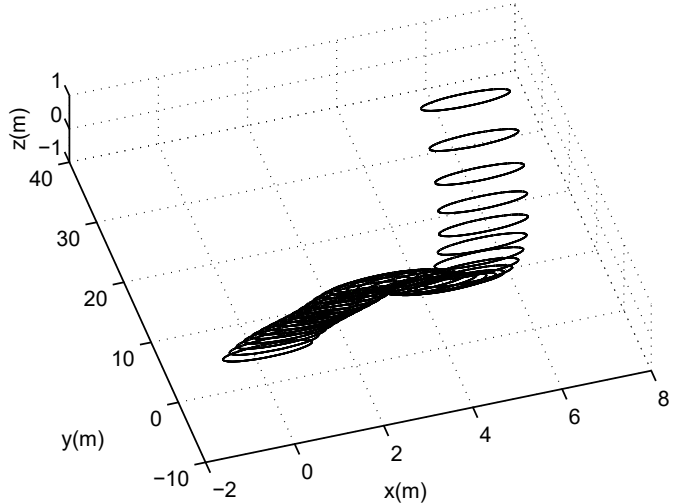

Figura 5: Ruta de seguridad volumétrica.

Módulo de Evitación de Obstáculos, define un camino fiable para evitar un impacto sobre el vehículo, determinado por la trayectoria proporcionada por el módulo de navegación y cualquier objeto que esté en la zona frontal del vehículo.

Se supone que cada objeto detectado es estático y se evalúa con las siguientes imágenes adquiridas para determinar si esta suposición es aceptada o rechazada. El siguiente análisis de imágenes adquiridas se realiza en dos etapas, en el primer 
paso, el módulo de evitación evalúa la posición relativa entre el vehículo y el objeto detectado $y$ en el segundo paso se verifica si se ha modificado la forma proporcional del objeto comprobando un umbral predefinido. En el caso afirmativo, el supuesto inicial es rechazado y el objeto se considera dinámico.

Después de esto, se calcula la trayectoria del objeto dinámico y se determina el tiempo de impacto. Tanto para objetos dinámicos como estáticos, el módulo de prevención de obstáculos calcula la nueva ruta y la envía al módulo de ejecución.

Módulo de búsqueda. Cuando se accede a este módulo, se carga la última posición de cable conocida y luego se establece un camino seguro hasta este punto, después de que el vehículo lo alcance, el algoritmo de cable de búsqueda establece una trayectoria cónica para el vehículo, comenzando desde el último punto conocido hasta el área teórica determinada por el módulo de navegación. Una vez determinado el cable de nuevo, el sistema elimina el conjunto de puntos correspondiente a la ruta anterior y el ciclo comienza de nuevo. En caso de no encontrar el vehículo, éste sube a la superficie y transmite la última posición conocida a la estación de control.

Módulo de ejecución se compone de dos módulos. Un módulo de decisión, en el que la acción a realizar se determina en cada momento. Es un sistema basado en eventos que depende del comportamiento del sistema. Si el módulo de prevención de obstáculos detecta un objeto, se genera un camino para evitar el objeto. El control vuelve al módulo de navegación una vez que los vehículos han evitado el objeto. Otro módulo corresponde al algoritmo de seguimiento de ruta, donde se aplica el método LOS (Line of Sight) modificado, cuyo objetivo es reducir el error transversal entre cada uno de los puntos obtenidos por los Módulos de Navegación y Evitación de Obstáculos.

\subsection{Características de la Arquitectura}

En la arquitectura propuesta se utiliza una plataforma multi-agente cooperativa, que consiste en un grupo de agentes que integran los conceptos de modularidad y escalabilidad requeridos para tales aplicaciones.

La modularidad de la arquitectura permite un aumento en la capacidad de intercambiabilidad y conectividad entre módulos evitando incompatibilidades y riesgos de entendimiento entre ellos.

La escalabilidad del sistema permite reducir errores, producidos por el cambio de nivel o entorno. Un aspecto de suma importancia es la necesidad de desarrollar diferentes estrategias de control operacional. Para ello se estima que se necesitará un módulo de verificación en el sistema. Por lo tanto, el módulo de generación de trayectorias se basa en datos previamente verificados y cuya interpretación no tiene inconsistencias potenciales que conduzcan respuestas indefinidas por el sistema de control. También se interpreta el trabajo de restricciones temporales de las capas, reduciendo los retrasos causados por los intérpretes de diferentes niveles en el lanzamiento de interrupciones o subrutinas para diferentes escenarios en el procesamiento de datos recibidos de sensores. El uso de esta arquitectura cooperativa multi-agente limita estos retrasos.

\section{Resultados de Simulación}

En base a lo expuesto en las secciones anteriores se ha implementado en el entorno Matlab/Simulink una máquina de estados finitos para la simulación del módulo de ejecución de navegación. Basado en un trabajo anterior [5], hemos implementado un modelo de maniobra para un sistema de guía basado en el método LOS modificado. Las corrientes oceánicas se han simulado mediante la inclusión de las fuerzas inducidas por la corriente y momentos en las ecuaciones dinámicas. Una forma de hacer esto es suponer que las ecuaciones están representadas en términos de la velocidad relativa. La forma en que se ha implementado la contribución de la corrientes al modelo de maniobra se encuentra en [4].

Los resultados para el sistema de guía se muestran en la figura 6 donde se comparan los resultados del LOS convencional con los de LOS modificado. Se observa una reducción en el error transversal de camino cuando se aplica el método LOS modificado para el vector de referencia de referencia dado por $([0,0 ; 20,25 ;-20,30 ;-30,40 ; 20,70 ; 0,80])$.

La figura 7 muestra la generación de trayectorias para el vehículo con forma de torpedo. La línea verde discontinua representa la trayectoria dada por el módulo de navegación, ya que se ve que está casi totalmente dentro del área delimitada por el rango de haz de detección de cable del SBL a una profundidad constante.

En la figura 8 se muestra el volumen de navegación fiable para varias profundidades mostradas en cada una de las posiciones alcanzables por el vehículo submarino dentro del rango de detección.

\section{Conclusiones}

En este artículo hemos propuesto una arquitectura basada en agentes múltiples que permite respon- 


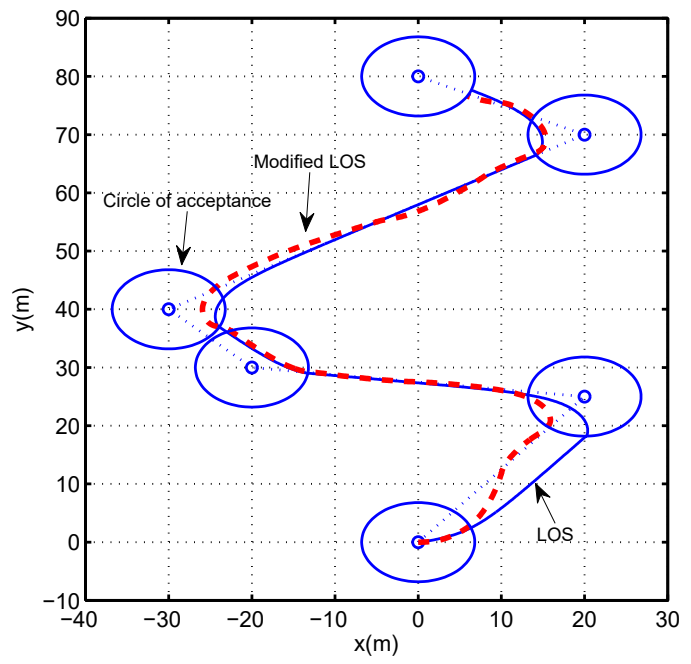

Figura 6: LOS convencional (línea azul) y LOS modificado (línea roja discontinua) con corrientes $(0,3 \mathrm{~m} / \mathrm{s})$ para el vector de referencia de waypoints $=[0,0 ; 20,25 ;-20,30 ;-30,40 ; 20,70 ; 0,80]$.

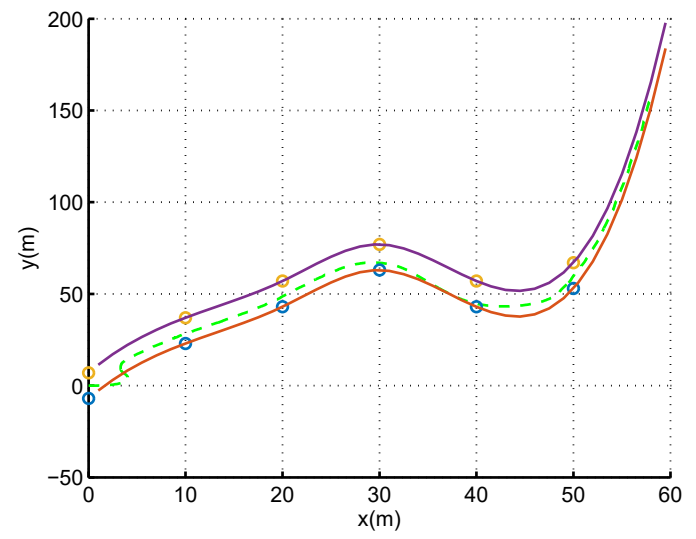

Figura 7: Área de detección (entre líneas azules y rojas) y camino simulado (línea verde discontinua) para el vehículo con forma de torpedo.

der a las restricciones de tiempo preestablecidas, que afectan tanto el tiempo como el seguimiento para la inspección de cables submarinos utilizando vehículos submarinos autónomos.

El uso de este tipo de vehículos en los últimos años se debe al avance en su autonomía, tanto desde el punto de vista de la energía como de la capacidad de decisión.

A través de la arquitectura propuesta, añadiendo la metodología basada en la generación de un espacio virtual tubular, perseguimos reducir la identificación del tiempo y mejorar el rendimiento de seguimiento del cable, además de dar una op-

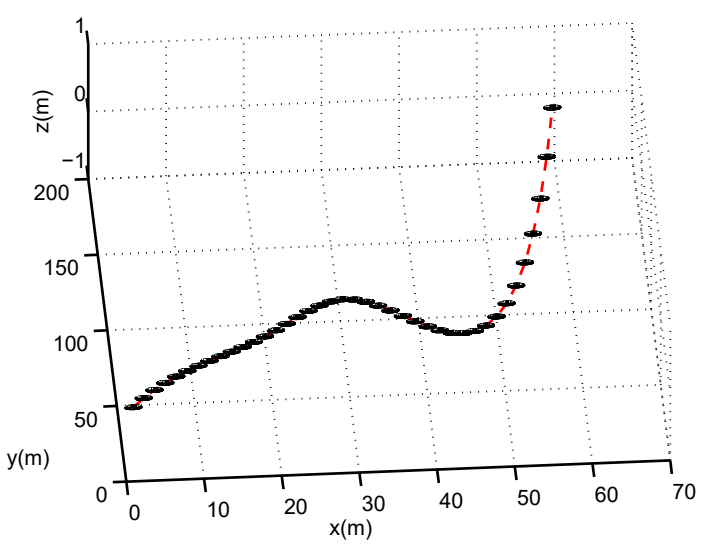

Figura 8: Volumen de detección y trayectoria desarrollada por el vehículo.

eración más fiable y segura.

\section{Agradecimientos}

Este trabajo ha sido parcialmente financiado por el MINECO: DPI2011-27990 con fondos FEDER.

\section{Referencias}

[1] Luntz Jonathan Almeida Emanuel and Tilbury Dawn. Modular finite state machines implemented as event-condition-action systems. In Proceedings of the 16th IFAC World Congress, 2005.

[2] K. Asakawa, J. Kojima, Y. Kato, S. Matsumoto, N. Kato, T. Asai, and T. Iso. Design concept and experimental results of the autonomous underwater vehicle aqua explorer 2 for the inspection of underwater cables. $A d$ vanced Robotics, 16(1):27 - 42, 2002.

[3] A. Bagnitsky, A. Inzartsev, A. Pavin, S. Melman, and M. Morozov. Side scan sonar using for underwater cables pipelines tracking by means of auv. pages 10 pp. -, Piscataway, NJ, USA, 2011//.

[4] T.I. Fossen. Marine Control Systems: Guidance, Navigation and Control of Ships, Rigs and Underwater Vehicles. Marine Cybernetics, 2002.

[5] Francisco J. Lastra Santos Jose Maria Riola Rodriguez Juan Jesus Diaz Hernandez Francisco J. Velasco, Elias Revestido Herrero and Luis M. Vega Antolin. Measurements of hydrodynamic parameters and control of an underwater torpedo-shaped vehicle. In IFAC Workshop on Navigation, Guidance and Control of Underwater Vehicles, 2015. 
[6] C.C. Insaurralde and D.M. Lane. Autonomyassessment criteria for underwater vehicles. pages 8 pp. -, Piscataway, NJ, USA, 2012.

[7] M. Jacobi and D. Karimanzira. Underwater pipeline and cable inspection using autonomous underwater vehicles. pages $6 \mathrm{pp}$.-, Piscataway, NJ, USA, 2013.

[8] Liviu Panait and Sean Luke. Cooperative multi-agent learning: The state of the art. Autonomous Agents and Multi-Agent Systems, 11(3):387-434, 2005.

[9] L. Pedraza, D. Rodriguez-Losada, F. Matia, G. Dissanayake, and J.V. Miro. Extending the limits of feature-based slam with b-splines. IEEE Transactions on Robotics, $25(2): 353-66,2009 /$.

[10] P.J. Ramadge and W.M. Wonham. Supervisory control of a class of discrete event processes. In A. Bensoussan and J.L. Lions, editors, Analysis and Optimization of Systems, volume 63 of Lecture Notes in Control and Information Sciences, pages 475-498. Springer Berlin Heidelberg, 1984.

[11] Stuart J. Russell and Peter Norvig. Artificial Intelligence: A Modern Approach. Pearson Education, 2 edition, 2003.

[12] Michael Woolridge and Michael J. Wooldridge. Introduction to Multiagent Systems. John Wiley \&amp; Sons, Inc., New York, NY, USA, 2001.

[13] O. Yildiz, A.E. Yilmaz, and B. Gokalp. State-of-the-art system solutions for unmanned underwater vehicles. Radioengineering, 18(4):590 - 600, 2009/12/. 